\title{
Telescopes get multimillion-dollar upgrades
}

A new generation of adaptive optics (AO) could give large ground-based telescopes an even clearer view of the cosmos. The W. M. Keck Observatory atop Mauna Kea in Hawaii is upgrading the AO systems on both of its $10 \mathrm{~m}$ telescopes Keck I and Keck II - thanks to funding from the National Science Foundation.

The US\$1.7 million grant is allowing researchers to design and build the first infrared tip-tilt sensor for the Keck I. Readings from the sensor will be used to control a fast mirror that corrects for atmospheric turbulence. The proposed upgrade will extend the operating spectrum of the Keck I from the visible to the near-infrared regime, thus improving the imaging of stars by $40-50 \%$. "This is a substantial gain in performance," says Tomasso Treu, principal scientist for the sensor. "It will have a huge scientific impact in certain areas of astronomy, such as in the study of distant galaxies."

A further grant of US\$1.3 million is allowing scientists to design a centrelaunch laser telescope that will be installed behind the secondary mirror of the Keck II. Light from the laser is absorbed and then re-emitted from the sodium layer in the atmosphere, forming an artificial guide star that functions as a reference signal. The AO system uses light from the guide star to measure

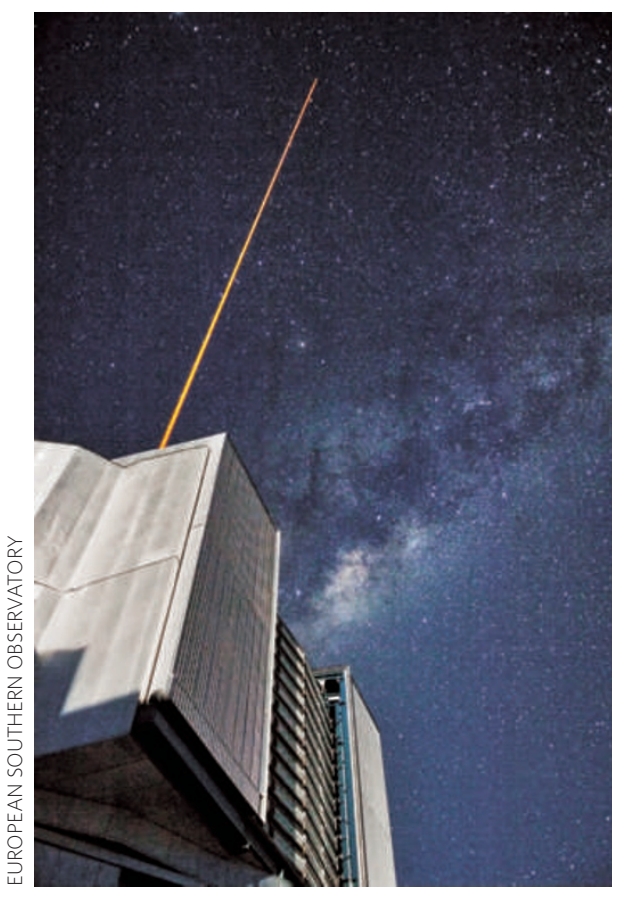

atmospheric turbulence and then deform the telescope's mirror to make appropriate corrections. The current laser is situated about a metre to one side of the telescope, which elongates the point of reflected light. Launching the laser from the centre of the telescope would reduce the measurement error and allow improved imaging. "We think we can improve the positional accuracy by a factor of two," says principal investigator Peter Wizinowich.

US\$3.8 million in funding is also being used to develop a seven-laser guide star system for Keck. This system is still in its design stage, however, with funding for the actual building yet to be approved. Although the launch system should be in place by 2011, the tip-tilt sensor could take around three years to be manufactured.

The European Southern Observatory has awarded $€ 5.2$ million to Toptica Photonics (Munich, Germany) and MPB Communications (Montreal, Canada) to build four laser guide stars for the Very Large Telescope in Chile. Previous guide stars have used either dye lasers or a mix of solid-state lasers, but these are inefficient and require significant maintenance. Toptica and MPB have now combined their technologies to design a narrowband Raman fibre amplifier that emits at $1,178 \mathrm{~nm}$ and uses resonanceenhanced frequency doubling to reach $589 \mathrm{~nm}$. Toptica says the lasers can produce $20 \mathrm{~W}$ of incident light at exactly the right wavelength for absorption by sodium, with a conversion efficiency of $80 \%$. The company expects the lasers to be installed on the Very Large Telescope within around three years.

\section{Laser fusion, planet finding and retinal imaging}

AO systems aren't just for large, groundbased telescopes. NASA has awarded two contracts worth US\$600,000 each to Boston Micromachines (Cambridge, Massachusetts, USA) to develop systems for the planned Terrestrial Planet Finder (TPF). The TPF, still in the conceptual stage, is a space-based system that will try to detect Earth-sized planets by measuring how much their gravitational pull affects the star they orbit.

One contract will fund the drive electronics required to move the actuators in the TPF's AO system, while the other will focus on fabricating $\mathrm{AO}$ mirror arrays comprising more than 3,000 actuators.

"They have to work perfectly, because there's no way to go up there and fix them," says Boston Micromachines CEO Paul Bierden.
Closer to Earth, the US Air Force Office of Scientific Research is funding the development of a new type of $\mathrm{AO}$ system that uses pairs of holograms as a wavefront sensor. By comparing how each half of the hologram pair affects the light passing through it, the sensor can measure the phase of the incident wavefront without a computer. Researcher Geoff Andersen says a simple wiring system would allow the actuators on a deformable mirror to be directly controlled by the detector elements themselves. Removing the computer makes the whole system lighter - an important consideration for unmanned aerial vehicles or airborne lasers.

Researchers at the Lawrence Livermore National Laboratory are exploring the use of a new AO interferometric system at the National Ignition Facility, which aims to achieve fusion by simultaneously slamming 192 high-power laser beams into a fuel target. Each of the beams must hit their target to within an accuracy of $\sim 100 \mu \mathrm{m}$. According to researchers, the new AO system should be capable of performing more than 10,000 beamsteering corrections per second - much faster than conventional AO.

US\$1.39 million in funding is also aiding research into the use of $\mathrm{AO}$ for analysing the human eye. Physical Sciences (Andover, Massachusetts, USA) has been awarded a two-year phase-II small business innovation research contract by the National Institutes of Health to develop a retinal imaging system that uses AO to perform simultaneous optical coherence tomography and scanning laser ophthalmoscope imaging. The system, being developed together with the University of Pittsburgh Medical Center, could potentially lead to earlier diagnosis or provide a better understanding of common eye diseases. 